\title{
Approximation by nonlinear integral operators in some modular function spaces
}

\author{
by Carlo Bardaro (Perugia), Julian Musielak (Poznań) \\ and Gianluca Vinti (Perugia)
}

\begin{abstract}
Let $G$ be a locally compact Hausdorff group with Haar measure, and let $L^{0}(G)$ be the space of extended real-valued measurable functions on $G$, finite a.e. Let $\varrho$ and $\eta$ be modulars on $L^{0}(G)$. The error of approximation $\varrho(a(T f-f))$ of a function $f \in\left(L^{0}(G)\right)_{\varrho+\eta} \cap \operatorname{Dom} T$ is estimated, where $(T f)(s)=\int_{G} K(t-s, f(t)) d t$ and $K$ satisfies a generalized Lipschitz condition with respect to the second variable.
\end{abstract}

1. Let $G$ be a locally compact Hausdorff group with neutral element $\theta$ and with the family $\mathcal{U}$ of open neighbourhoods of $\theta$ in $G$. For the sake of simplicity from now on we will assume $G$ to be abelian. Let $\Sigma$ be the Borel $\sigma$-field of $G$, let $|A|$ be the Haar measure of a measurable set $A \subset G$ and let $\int_{G} f(t) d t$ denote the Haar integral of $f$.

We shall denote by $M^{0}(G)$ the space of all extended real-valued measurable functions $f: G \rightarrow \overline{\mathbb{R}}=\mathbb{R} \cup\{\mp \infty\}$, and by $L^{0}(G) \subset M^{0}(G)$ its subspace of functions $f$ finite almost everywhere (a.e.), both provided with equality a.e.

Let $\varrho: L^{0}(G) \rightarrow \overline{\mathbb{R}}_{0}^{+}$and $\eta: L^{0}(G) \rightarrow \overline{\mathbb{R}}_{0}^{+}$be two modulars in $L^{0}(G)$, and let $\left(L^{0}(G)\right)_{\varrho}$ and $\left(L^{0}(G)\right)_{\eta}$ be the respective modular spaces (for terminology, see e.g. [4]).

We make the following assumptions:

$1^{\circ} \varrho$ and $\eta$ are monotone, i.e. if $f, g \in L^{0}(G)$ and $|f| \leq|g|$, then $\varrho(f) \leq$ $\varrho(g)$ and $\eta(f) \leq \eta(g)$;

$2^{\circ} \varrho$ is $\mathcal{J}$-convex, i.e. for any two measurable functions $p: G \rightarrow \mathbb{R}_{0}^{+}$and

1991 Mathematics Subject Classification: 46E30,46A80,41A46,45P05.

Key words and phrases: modular space, nonlinear integral operator, generalized Lipschitz condition, approximation by singular integrals.

This paper was written when the second author was a Visiting Professor at the Dipartimento di Matematica, Università degli Studi di Perugia. 
$F: G \times G \rightarrow \mathbb{R}$ with $\int_{G} p(t) d t=1$,

$$
\varrho\left(\int_{G} p(t)|F(t, \cdot)| d t\right) \leq \int_{G} p(t) \varrho(F(t, \cdot)) d t
$$

$3^{\circ} \eta$ is $\tau$-bounded, i.e. there are a number $c \geq 1$ and a measurable, bounded function $h: G \rightarrow \mathbb{R}_{0}^{+}$such that $h(t) \rightarrow 0$ as $t \rightarrow \theta$ and

$$
\eta(f(t+\cdot)) \leq \eta(c f)+h(t), \quad t \in G,
$$

for all $f \in L^{0}(G)$ such that $\eta(f)<\infty$; we shall write $h_{0}=\sup _{t \in G} h(t)$.

We may extend both modulars $\varrho$ and $\eta$ to $M^{0}(G)$, putting $\varrho(f)$ $=\eta(f)=\infty$ for $f \in M^{0}(G) \backslash L^{0}(G)$.

Let $\psi: G \times \mathbb{R}_{0}^{+} \rightarrow \mathbb{R}_{0}^{+}$be such that for all $t \in G$, the function $\psi(t, \cdot)$ is continuous and nondecreasing for $u \geq 0, \psi(t, 0)=0, \psi(t, u)>0$ for $u>0, \psi(t, u) \rightarrow \infty$ as $u \rightarrow \infty$, and such that for every $u \geq 0, \psi(t, u)$ is a measurable function of $t$.

The following connection between both modulars $\varrho$ and $\eta$ and the function $\psi$ will be assumed:

(I) there is a set $G_{0} \subset G$ with $\left|G \backslash G_{0}\right|=0$ such that for every $\left.\lambda \in\right] 0,1[$ there exists a $\left.C_{\lambda} \in\right] 0,1[$ satisfying the inequality

$$
\varrho\left[C_{\lambda} \psi(t,|F(\cdot)|)\right] \leq \eta(\lambda F(\cdot))
$$

for all $t \in G_{0}$ and $F \in L^{0}(G)$.

A condition of this type was introduced in special cases in [3].

Let us still remark that we may choose $C_{\lambda}$ in such a manner that $C_{\lambda} \searrow 0$ as $\lambda \searrow 0$. Condition (I) implies immediately the following inequality:

$$
\varrho\left[C_{\lambda} \psi\left(t, F_{t}(\cdot)\right)\right] \leq \eta\left(\lambda F_{t}(\cdot)\right)
$$

for every $t \in G_{0}$ and for any family $\left(F_{t}(\cdot)\right)_{t \in G}$ of functions $F_{t} \in L^{0}(G)$.

A function $K: G \times \mathbb{R} \rightarrow \mathbb{R}$ will be called a kernel function if $K(t, 0)=0$ for $t \in G$ and $K(\cdot, u) \in L^{1}(G)$ for all $u \in \mathbb{R}$. Let $L: G \rightarrow \mathbb{R}_{0}^{+}, L \in L^{1}(G)$. We say that a kernel function $K$ satisfies the $(L, \psi)$-Lipschitz condition if

$$
|K(t, u)-K(t, v)| \leq L(t) \psi(t,|u-v|)
$$

for $t \in G, u, v \in \mathbb{R}$ (see [1], p. 10).

In the following we shall write $L=\int_{G} L(t) d t, p(t)=L(t) / L$.

Let us remark that if $K$ is an $(L, \psi)$-Lipschitz kernel function and $f \in L^{0}(G)$, then the superposition $K(t, f(t+s))$ is a measurable function of $t \in G$ for all $s \in G$.

2. Following $[1-4]$ we shall deal with nonlinear integral operators $T$ of the form 


$$
(T f)(s)=\int_{G} K(t-s, f(t)) d t=\int_{G} K(t, f(t+s)) d t .
$$

We denote by $\operatorname{Dom} T$ the set of all functions $f \in L^{0}(G)$ such that $(T f)(s)$ exists for a.e. $s \in G$ and $T f$ is a measurable function on $G$.

Proposition 1. Let $f \in\left(L^{0}(G)\right)_{\eta} \cap \operatorname{Dom} T$ and let $\left.\lambda \in\right] 0,1[$ be so small that $\eta(c \lambda f)<\infty$, where $c \geq 1$ is the constant from $3^{\circ}$. Suppose that $K$ is an $(L, \psi)$-Lipschitz kernel function and the condition (I) is satisfied. Then, for every $\varepsilon>0$, there exists a $U \in \mathcal{U}$ such that

$$
\varrho\left(\frac{C_{\lambda}}{L} T f\right) \leq \eta(c \lambda f)+h_{0} \int_{G \backslash U} p(t) d t+\varepsilon .
$$

Consequently, $\varrho\left(\left(C_{\lambda} / L\right) T f\right)<\infty$.

Proof. Applying monotonicity of $\varrho$, the $(L, \psi)$-Lipschitz condition, $\mathcal{J}$-convexity of $\varrho$ and the condition (I), we obtain

$$
\begin{aligned}
\varrho\left(\frac{C_{\lambda}}{L} T f\right) & \leq \varrho\left(\frac{C_{\lambda}}{L} \int_{G} \mid K(t, f(t+\cdot) \mid) d t\right) \leq \varrho\left(\int_{G} p(t) C_{\lambda} \psi(t,|f(t+\cdot)|) d t\right) \\
& \leq \int_{G} p(t) \varrho\left[C_{\lambda} \psi(t,|f(t+\cdot)|)\right] d t \leq \int_{G} p(t) \eta(\lambda|f(t+\cdot)|) d t .
\end{aligned}
$$

Since $\eta(\lambda f)<\infty$, by $\tau$-boundedness of $\eta$, we get $\eta(\lambda|f(t+\cdot)|) \leq \eta(c \lambda f)+h(t)$ for $t \in G$. Consequently, since $\int_{G} p(t) d t=1$, we obtain

$$
\varrho\left(\frac{C_{\lambda}}{L} T f\right) \leq \eta(c \lambda f)+\int_{G} p(t) h(t) d t .
$$

However, since $h(t) \rightarrow 0$ as $t \rightarrow \theta$, for any $\varepsilon>0$ there is a $U \in \mathcal{U}$ such that $h(t)<\varepsilon$ for $t \in U$. Since $h(t) \leq h_{0}$ for $t \in G \backslash U$, the required inequality follows from (1).

3. The map $\omega_{\eta}: L^{0}(G) \times \mathcal{U} \rightarrow \overline{\mathbb{R}}_{0}^{+}=[0, \infty]$ defined by

$$
\omega_{\eta}(f, U)=\sup _{t \in U} \eta(f(t+\cdot)-f(\cdot))
$$

for $f \in L^{0}(G), U \in \mathcal{U}$, is called the $\eta$-modulus of continuity (see [4], p. 85). We shall apply the following notation:

$$
\begin{gathered}
r_{k}=\sup _{1 / k \leq|u| \leq k}\left|\frac{1}{u} \int_{G} K(t, u) d t-1\right|, \quad A_{k}=\{t \in G:|f(t)|>k\}, \\
B_{k}=\{t \in G:|f(t)|<1 / k\}, \quad C_{k}=G \backslash\left(A_{k} \cup B_{k}\right), \quad f \in L^{0}(G), \\
k=1,2, \ldots, \quad \text { and } r=\sup _{k} r_{k} .
\end{gathered}
$$


We shall give an estimate of the modular error of approximation $\varrho(a(T f-f))$ for sufficiently small $a>0$.

Theorem 1. Let $f \in\left(L^{0}(G)\right)_{\varrho+\eta} \cap \operatorname{Dom} T$. Let $\left.\lambda \in\right] 0,1[$ and $a \in$ ] $0, C_{\lambda} /(16 L)$ [ be so small that $\eta(2 c \lambda f)<\infty$ and $\varrho(16 a f)<\infty$. Then, for every $U \in \mathcal{U}, k=0,1,2, \ldots$ and $S \in \Sigma$, we have

(2) $\varrho(a(T f-f)) \leq \omega_{\eta}(\lambda f, U)+\left[2 \eta(2 c \lambda f)+h_{0}\right] \int_{G \backslash U} p(t) d t+R_{k}$,

where $R_{k}$ is given by

$$
\begin{aligned}
R_{0}= & \varrho(2 a r f), \\
R_{k}= & \eta\left(\lambda f \chi_{G \backslash S}\right)+\varrho\left(16 a f \chi_{G \backslash S}\right)+\eta\left(\lambda f \chi_{S \cap A_{k}}\right) \\
& +\varrho\left(16 a f \chi_{S \cap A_{k}}\right)+\eta\left(\lambda f \chi_{S \cap B_{k}}\right)+\varrho\left(16 a f \chi_{S \cap B_{k}}\right) \\
& +\varrho\left(\text { gar }_{k} f\right), \quad k=1,2, \ldots
\end{aligned}
$$

Pro of. We have $\varrho(a(T f-f)) \leq J_{1}+J_{2}$, where

$$
\begin{aligned}
& J_{1}=\varrho\left\{2 a \int_{G}|K(t, f(t+\cdot))-K(t, f(\cdot))| d t\right\}, \\
& J_{2}=\varrho\left\{2 a\left|\int_{G} K(t, f(\cdot)) d t-f(\cdot)\right|\right\}
\end{aligned}
$$

(see [4], p. 88). By the $(L, \psi)$-Lipschitz condition, by $\mathcal{J}$-convexity of $\varrho$ and taking into account the condition (I) we have

$$
\begin{aligned}
J_{1} \leq & \int_{G} p(t) \varrho\left[C_{\lambda} \psi(t,|f(t+\cdot)-f(\cdot)|] d t\right. \\
\leq & \int_{U} p(t) \eta(\lambda|f(t+\cdot)-f(\cdot)|) d t \\
& \quad+\int_{G \backslash U} p(t) \eta(\lambda|f(t+\cdot)-f(\cdot)|) d t=J_{1}^{1}+J_{1}^{2} .
\end{aligned}
$$

But

$$
J_{1}^{1} \leq \int_{G} p(t) \omega_{\eta}(\lambda f, U) d t \leq \omega_{\eta}(\lambda f, U)
$$

Now,

$$
J_{1}^{2} \leq \int_{G \backslash U} p(t) \eta(2 \lambda f(t+\cdot)) d t+\eta(2 \lambda f) \int_{G \backslash U} p(t) d t .
$$

By $\tau$-boundedness of $\eta$ we obtain

$$
\int_{G \backslash U} p(t) \eta(2 \lambda f(t+\cdot)) d t \leq\left[\eta(2 c \lambda f)+h_{0}\right] \int_{G \backslash U} p(t) d t
$$


whence, by monotonicity of $\eta$,

$$
J_{1}^{2} \leq\left[2 \eta(2 c \lambda f)+h_{0}\right] \int_{G \backslash U} p(t) d t .
$$

Consequently,

$$
J_{1} \leq \omega_{\eta}(\lambda f, U)+\left[2 \eta(2 c \lambda f)+h_{0}\right] \int_{G \backslash U} p(t) d t .
$$

It remains to prove that $J_{2} \leq R_{k}, k=0,1,2, \ldots$ For $k=0$ this is obvious. Suppose $k>0$. Then, taking any set $S \in \Sigma$, we have

$$
\begin{aligned}
J_{2} \leq & \varrho\left\{8 a\left|\int_{G} K\left(t, f(\cdot) \chi_{G \backslash S}(\cdot)\right) d t-f(\cdot) \chi_{G \backslash S}(\cdot)\right|\right\} \\
& +\varrho\left\{8 a\left|\int_{G} K\left(t, f(\cdot) \chi_{S \cap A_{k}}(\cdot)\right) d t-f(\cdot) \chi_{S \cap A_{k}}(\cdot)\right|\right\} \\
& +\varrho\left\{8 a\left|\int_{G} K\left(t, f(\cdot) \chi_{S \cap B_{k}}(\cdot)\right) d t-f(\cdot) \chi_{S \cap B_{k}}(\cdot)\right|\right\} \\
& +\varrho\left\{8 a\left|\int_{G} K\left(t, f(\cdot) \chi_{S \cap C_{k}}(\cdot)\right) d t-f(\cdot) \chi_{S \cap C_{k}}(\cdot)\right|\right\} .
\end{aligned}
$$

By $(L, \psi)$-Lipschitz condition, monotonicity and $\mathcal{J}$-convexity of $\varrho$ and by condition (I), for every $P \in \Sigma$ we get

$$
\begin{aligned}
\varrho\left\{8 a \mid \int_{G} K\left(t, f(\cdot) \chi_{P}(\cdot)\right)\right. & \left.d t-f(\cdot) \chi_{P}(\cdot) \mid\right\} \\
\leq & \varrho\left\{16 a \int_{G}\left|K\left(t, f(\cdot) \chi_{P}(\cdot)\right)\right| d t\right\}+\varrho\left(16 a f \chi_{P}\right) \\
& \leq \int_{G} p(t) \varrho\left[C_{\lambda} \psi\left(t, f(\cdot) \chi_{P}(\cdot)\right)\right] d t+\varrho\left(16 a f \chi_{P}\right) \\
& \leq \eta\left(\lambda f \chi_{P}\right)+\varrho\left(16 a f \chi_{P}\right) .
\end{aligned}
$$

Thus, for $P=G \backslash S, P=S \cap A_{k}, P=S \cap B_{k}$ and by the definition of $r_{k}$, we obtain

$$
\begin{aligned}
J_{2} \leq & \eta\left(\lambda f \chi_{G \backslash S}\right)+\varrho\left(16 a f \chi_{G \backslash S}\right)+\eta\left(\lambda f \chi_{S \cap A_{k}}\right)+\varrho\left(16 a f \chi_{S \cap A_{k}}\right) \\
& +\eta\left(\lambda f \chi_{S \cap B_{k}}\right)+\varrho\left(16 a f \chi_{S \cap B_{k}}\right)+\varrho\left(8 a r_{k} f\right)
\end{aligned}
$$

and so the assertion follows.

4. Let $\mathcal{W}$ be a nonempty, abstract set of indices and let $\mathbf{W}$ be a filter of subsets of $\mathcal{W}$.

A family $\mathbb{K}=\left(K_{w}\right)_{w \in \mathcal{W}}$ of kernel functions will be called a kernel. 
Let $\mathbb{L}=\left(L_{w}\right)_{w \in \mathcal{W}}$ be a family of nonnegative functions $L_{w} \in L^{1}(G)$. We say that the kernel $\mathbb{K}$ satisfies the $(\mathbb{L}, \psi)$-Lipschitz condition if the kernel functions $K_{w}$ satisfy the $\left(L_{w}, \psi\right)$-Lipschitz condition, and $L=\sup _{\mathcal{W}} L_{w}=$ $\sup _{\mathcal{W}} \int_{G} L_{w}(t) d t<\infty$. Set $p_{w}(t)=L_{w}(t) / L_{w}$ (see [1], pp. 12-13). The kernel $\mathbb{K}$ will be called singular if for every $U \in \mathcal{U}$,

$$
\int_{G \backslash U} p_{w}(t) d t \stackrel{\mathbf{W}}{\longrightarrow} 0
$$

and

$$
r_{k}(w)=\sup _{1 / k \leq|u| \leq k}\left|\frac{1}{u} \int_{G} K_{w}(t, u) d t-1\right| \stackrel{\mathbf{W}}{\longrightarrow} 0
$$

for $k=1,2, \ldots$ If, moreover,

$$
r(w)=\sup _{k=1,2, \ldots} r_{k}(w) \stackrel{\mathbf{W}}{\longrightarrow} 0,
$$

the kernel $\mathbb{K}$ will be called strongly singular.

Let us define a family $\mathbb{T}=\left(T_{w}\right)_{w \in \mathcal{W}}$ of operators by

$$
\left(T_{w} f\right)(s)=\int_{G} K_{w}(t-s, f(t)) d t=\int_{G} K_{w}(t, f(t+s)) d t .
$$

Set Dom $\mathbb{T}=\bigcap_{w \in \mathcal{W}} \operatorname{Dom} T_{w}$. We shall deduce from Theorem 1 a theorem on convergence $\varrho\left(a\left(T_{w} f-f\right)\right) \stackrel{\mathbf{W}}{\longrightarrow} 0$ for small $a>0$. We need some additional notions, namely of absolute finiteness and absolute continuity of modulars (see [4], p. 84, [2], p. 4).

Definition 1. A modular $\eta$ on $L^{0}(G)$ is called finite if for every measurable set $A \subset G$ such that $|A|<\infty$ we have $\chi_{A} \in\left(L^{0}(G)\right)_{\eta}$.

DEFINITION 2. A modular $\eta$ on $L^{0}(G)$ is called absolutely finite if it is finite and if for every $\varepsilon>0$ and for every $\lambda_{0}>0$, there is a $\delta>0$ such that $\eta\left(\lambda_{0} \chi_{B}\right)<\varepsilon$ for every measurable set $B \subset G$ of measure $|B|<\delta$.

Definition 3. A modular $\eta$ on $L^{0}(G)$ is called absolutely continuous (with respect to the measure in $G$ ) if there exists an $\alpha>0$ such that for every $f \in L^{0}(G)$ with $\eta(f)<\infty$ the following two conditions are satisfied:

$(\alpha)$ for every $\varepsilon>0$ there exists a measurable set $A \subset G$ such that $|A|<\infty$ and $\eta\left(\alpha f \chi_{G \backslash A}\right)<\varepsilon$

$(\beta)$ for every $\varepsilon>0$ there exists a $\delta>0$ such that $\eta\left(\alpha f \chi_{B}\right)<\varepsilon$ for all measurable sets $B \subset G$ of measure $|B|<\delta$.

Let us remark that if $\eta$ is monotone, $\tau$-bounded, absolutely finite and absolutely continuous, then for every $f \in\left(L^{0}(G)\right)_{\eta}$ there is a $\lambda_{0}>0$ such that for every $\varepsilon>0$ there exists a $U_{\varepsilon} \in \mathcal{U}$ such that $\omega_{\eta}\left(\lambda_{0} f, U_{\varepsilon}\right)<\varepsilon$ (see 
[4], Theorem 1, p. 85; the condition (P) mentioned there is always satisfied, as was kindly shown to us by Prof. D. Candeloro).

THEOREM 2. Let $\mathbb{K}=\left(K_{w}\right)_{w \in \mathcal{W}}$ be a singular kernel and let the modular $\varrho$ be monotone and $\mathcal{J}$-convex, and the modular $\eta$-monotone, $\tau$-bounded, absolutely finite and absolutely continuous. Let $f \in\left(L^{0}(G)\right)_{\varrho+\eta} \cap \operatorname{Dom} \mathbb{T}$. Finally, let one of the following conditions hold:

(a) $\mathbb{K}$ is strongly singular;

(b) @ is finite and absolutely continuous.

Then $\varrho\left(a\left(T_{w} f-f\right)\right) \stackrel{\mathbf{w}}{\longrightarrow} 0$ for sufficiently small $a>0$ (depending on $\left.f\right)$.

Proof. Choose an arbitrary $\varepsilon>0$. Since $\eta$ is monotone, $\tau$-bounded, absolutely finite and absolutely continuous, there is a $U \in \mathcal{U}$ such that $\omega_{\eta}\left(\lambda_{0} f, U\right)<\varepsilon / 4$ for sufficiently small $\lambda_{0}>0$. Taking $\left.\lambda_{1} \in\right] 0, \lambda_{0}$ [ small enough, we get $\eta\left(2 c \lambda_{1} f\right)<\infty$. Due to singularity of $\mathbb{K}$, keeping the above $U \in \mathcal{U}$ fixed, we have $\int_{G \backslash U} p_{w}(t) d t \stackrel{\mathbf{W}}{\longrightarrow} 0$. Hence there exists a $\mathcal{W}_{1} \in \mathbf{W}$ such that

$$
\left[2 \eta(2 c \lambda f)+h_{0}\right] \int_{G \backslash U} p_{w}(t) d t<\varepsilon / 4
$$

for $\left.\lambda \in] 0, \lambda_{1}\right], w \in \mathcal{W}_{1}$ and the above $U \in \mathcal{U}$.

Thus, for a fixed $\lambda \in] 0, \lambda_{1}$ [ let $C_{\lambda}$ be the corresponding constant in (I), and for $a \in] 0, C_{\lambda} /(16 L)[$, we have

$$
\varrho\left(a\left(T_{w} f-f\right)\right)<\varepsilon / 2+R_{k}
$$

for $w \in \mathcal{W}_{1}$ and $k=0,1,2, \ldots$, where we have applied (2) with $T_{w}$ and $p_{w}$ in place of $T$ and $p$.

Assuming that (a) holds, we apply (3) with $k=0$, obtaining $R_{0}=$ $\varrho(2 a r(w) f)$. However, since $f \in\left(L^{0}(G)\right)_{\varrho}$, there is a $\mathcal{W}_{2} \in \mathbf{W}$ such that $\varrho(2 a r(w) f)<\varepsilon / 2$ for $w \in \mathcal{W}_{2}$. This gives $\varrho\left(a\left(T_{w} f-f\right)\right)<\varepsilon$ for $w \in$ $\mathcal{W}_{1} \cap \mathcal{W}_{2} \in \mathbf{W}$, which implies our assertion.

Now suppose (b). We apply Theorem 1 with a given $S \in \Sigma$ with $|S|<\infty$. Since $A_{1} \supset A_{2} \supset \ldots$, we have $S \cap A_{1} \supset S \cap A_{2} \supset \ldots$, and $\left|S \cap A_{1}\right|<\infty$. Hence $\lim _{k \rightarrow \infty}\left|S \cap A_{k}\right|=\left|S \cap \bigcap_{k=1}^{\infty} A_{k}\right|$. But $f \in L^{0}(G)$ whence there exists a set $G_{0} \subset G, G_{0} \in \Sigma,\left|G_{0}\right|=0$, such that $|f(t)|<\infty$ for $t \in G \backslash G_{0}$. From the inclusion $\bigcap_{k=1}^{\infty} A_{k} \subset G_{0}$, we deduce $\lim _{k \rightarrow \infty}\left|S \cap A_{k}\right|=0$.

Now applying absolute continuity of $\eta$ and $\varrho$, we may choose $\lambda$ and $a$ so small that

$$
\eta\left(\lambda f \chi_{G \backslash S}\right)+\varrho\left(16 a f \chi_{G \backslash S}\right)<\varepsilon / 12
$$

for a suitable set $S \in \Sigma,|S|<\infty$.

Keeping $S$ fixed, we may find an index $k$ such that

$$
\eta\left(\lambda f \chi_{S \cap A_{k}}\right)+\varrho\left(16 a f \chi_{S \cap A_{k}}\right)<\varepsilon / 12 .
$$


Moreover, $\eta\left(\lambda f \chi_{S \cap B_{k}}\right)+\varrho\left(16 a f \chi_{S \cap B_{k}}\right) \leq \eta\left((\lambda / k) \chi_{S}\right)+\varrho\left((16 a / k) \chi_{S}\right)$ and since $\chi_{S} \in\left(L^{0}(G)\right)_{\varrho+\eta}$ we may find $k$ such that $\eta\left(\lambda f \chi_{S \cap B_{k}}\right)+\varrho\left(16 a f \chi_{S \cap B_{k}}\right)$ $<\varepsilon / 12$, which gives $R_{k}<\varepsilon / 4+\varrho\left(\operatorname{8ar}_{k}(w) f\right)$. Taking $w \in \mathcal{W}_{1}$ we obtain by $(3), \varrho\left(a\left(T_{w} f-f\right)\right)<3 \varepsilon / 4+\varrho\left(8 a r_{k}(w) f\right)$. But $f \in\left(L^{0}(G)\right)_{\varrho}$, whence there is a $\mathcal{W}_{3} \in \mathbf{W}$ such that $\varrho\left(8 a r_{k}(w) f\right)<\varepsilon / 4$ for $w \in \mathcal{W}_{3}$. This gives $\varrho\left(a\left(T_{w} f-f\right)\right)<\varepsilon$ for $w \in \mathcal{W}_{1} \cap \mathcal{W}_{3} \in \mathbf{W}$, which implies our statement.

5. We give some examples of modulars $\varrho$ and $\eta$ satisfying the assumptions of Theorems 1 and 2 .

EXAMPLES. 1. Let $\Phi: \mathbb{R}_{0}^{+} \rightarrow \mathbb{R}_{0}^{+}$be such that $\Phi(0)=0, \Phi(u)>0$ for $u>0, \Phi$ nondecreasing in $\mathbb{R}_{0}^{+}$, and $\Phi(u) \rightarrow \infty$ as $u \rightarrow \infty$. Then $\Phi$ generates a modular

$$
\sigma(f)=I_{\Phi}(f)=\int_{G} \Phi(|f(t)|) d t
$$

in $L^{0}(G)$, and the respective modular space $\left(L^{0}(G)\right)_{\sigma}$ is the Orlicz space $L^{\Phi}(G)$.

The modular $\sigma$ is monotone, absolutely finite, absolutely continuous and $\tau$-bounded (with $c=1, h(t) \equiv 0$ ). If $\Phi$ is convex on $\mathbb{R}_{0}^{+}$, then $\sigma$ is $\mathcal{J}$-convex. Thus, $I_{\Phi}$ satisfies the assumption of Theorem 2 , (b).

Finally, if we take two functions $\Phi_{1}$ and $\Phi_{2}$ and we put $\varrho=I_{\Phi_{1}}, \eta=I_{\Phi_{2}}$, then (I) is certainly satisfied with $\lambda=C_{\lambda}$ if we assume the concavity of the function $\psi$ with respect to the second variable and that $\left(\Phi_{1} \circ \psi\right)(u) \leq \Phi_{2}(u)$ for $u \geq 0$.

2. Let $\mathcal{V}$ be a nonempty set of indices filtered by a set $\mathbf{W}$ of its subsets. Let $a_{v}:\left[a, b\left[\rightarrow \mathbb{R}_{0}^{+}, v \in \mathcal{V}\right.\right.$, be such that

$1^{\circ} \int_{a}^{b} a_{v}(x) d x \leq 1$ for all $v \in \mathcal{V}$;

$2^{\circ}$ if $g:\left[a, b\left[\rightarrow \mathbb{R}_{0}^{+}\right.\right.$is such that $0 \leq g(x) \nearrow s<\infty$ as $x \rightarrow b-$, then

$$
\int_{a}^{b} a_{v}(x) g(x) d m \stackrel{\mathbf{W}}{\longrightarrow} s ;
$$

$3^{\circ}$ for every Lebesgue measurable set $C \subset[a, b[$ of measure $m(C)>0$ there exists a Lebesgue measurable subset $C_{1}$ of measure $m\left(C_{1}\right)>0$ and an index $\bar{v} \in \mathcal{V}$ such that $a_{\bar{v}}(x)>0 m$-almost everywhere in $C_{1}$.

Let $\Phi:\left[a, b\left[\times \mathbb{R}_{0}^{+} \rightarrow \mathbb{R}_{0}^{+}\right.\right.$satisfy

1) $\Phi(x, u)$ is a nondecreasing continuous function of $u \geq 0$, for every $x \in[a, b[$;

2) $\Phi(x, 0)=0, \Phi(x, u)>0$ for $u>0$, and $\Phi(x, u) \rightarrow \infty$ as $u \rightarrow \infty$, for every $x \in[a, b[$; 
3) the $\operatorname{limit}_{\lim _{x \rightarrow b-}} \Phi(x, u)=\widetilde{\Phi}(u)<\infty$ exists for every $u \geq 0$;

4) $\Phi(x, u)$ is a Lebesgue measurable function of $x$ in $[a, b[$, for every $u \geq 0$.

Moreover, suppose $\Phi(x, u)$ to be of monotone type in a subinterval $[c, b[\subset$ $[a, b[$ and equicontinuous in $[a, b[$ at $u=0$ (for these notions see [2], Section 4).

Let $m$ be a measure on $[a, b[$ defined on all Lebesgue measurable subsets of $[a, b[$. Then

$$
\mathcal{A}_{\Phi}(f)=\sup _{v \in \mathcal{V}} \int_{a}^{b} a_{v}(x) \mathcal{J}_{\Phi}(x, f) d m(x),
$$

where

$$
\mathcal{J}_{\Phi}(x, f)=\int_{G} \Phi(x,|f(t)|) d t,
$$

is a modular in the subspace $L_{m}^{0}(G) \subset L^{0}(G)$ of functions $f$ for which $\mathcal{J}_{\Phi}(x, f)$ is a Lebesgue measurable function on $[a, b[$.

In [2] sufficient conditions are obtained in order that $\mathcal{A}_{\Phi}$ be absolutely finite and absolutely continuous. Evidently $\mathcal{A}_{\Phi}$ is monotone. If $\Phi(x, u)$ is a convex function of $u \geq 0$ for all $x \in[a, b[$, then for all measurable functions $F: G \times G \rightarrow \mathbb{R}$ and $p: G \rightarrow \mathbb{R}_{0}^{+}$with $\int_{G} p(t) d t=1$ we have Jensen's inequality

$$
\Phi\left(x, \int_{G} p(t) F(t, s) d t\right) \leq \int_{G} p(t) \Phi(x,|F(t, s)|) d t
$$

for $x \in\left[a, b\left[, s \in G\right.\right.$. Hence it follows that, in this case, $\mathcal{A}_{\Phi}$ is $\mathcal{J}$-convex. It is easily observed that $\mathcal{J}_{\Phi}(x, f(t+\cdot))=\mathcal{J}_{\Phi}(x, f)$ for every $t \in G$, whence $\mathcal{A}_{\Phi}$ is $\tau$-bounded with $c=1, h(t) \equiv 0$.

The theory developed in [2] for the modular $\mathcal{A}_{\Phi}$ contains as a particular case the discrete modulars of the type

$$
\mathcal{A}_{\Phi}(f)=\sup _{n \in \mathbb{N}} \sum_{i=1}^{\infty} a_{n, i} I_{\Phi_{i}}(f)
$$

(for details see [2], Section 5).

Moreover, it is possible to prove that the more general modulars of type

$$
\widetilde{\mathcal{A}}_{\Phi}(f)=\sup _{v \in \mathcal{V}} \int_{a}^{b} \mathcal{J}_{\Phi}(x, f) d m_{w}(x), \quad f \in L_{m}^{0}(G),
$$

where $\left\{m_{w}\right\}$ is a family of measures, satisfy the conditions of Theorem 1 and Theorem 2, under the assumptions of Section 6 of [2]. 
We remark that among these modulars there are those studied in [6], namely

$$
I_{\Phi}(f)=\sup _{x \in[a, b[} \mathcal{J}_{\Phi}(x, f)
$$

(see also Section 6 of [2]).

Hence we may state the following

Corollary 1 . Let $\mathbb{K}=\left(K_{w}\right)_{w \in \mathcal{W}}$ be a singular kernel and let $\varrho, \eta$ be any of the modulars defined in Examples 1 or 2, satisfying (I). Suppose the function $\Phi$ generating $\varrho$ is convex. In case any of the modulars is as in Example 2, suppose that the respective function $\Phi$ satisfies the assumptions of Theorem 1 of [2]. Then, for any function $f \in\left(L^{0}(G)\right)_{\varrho+\eta} \cap \operatorname{Dom} \mathbb{T}$,

$$
\varrho\left(a\left(T_{w} f-f\right)\right) \stackrel{\mathbf{W}}{\longrightarrow} 0
$$

for sufficiently small $a>0$.

\section{References}

[1] C. Bardaro, J. Musielak and G. Vinti, Modular estimates and modular convergence for a class of nonlinear operators, Math. Japon. 39 (1994), 7-14.

[2] - - - - - On absolute continuity of a modular connected with strong summability, Comment. Math. Prace Mat. 34 (1994), 21-33.

[3] C. Bardaro and G. Vinti, Modular approximation by nonlinear integral operators on locally compact groups, ibid., to appear.

[4] J. Musielak, Nonlinear approximation in some modular function spaces. I, Math. Japon. 38 (1993), 83-90.

[5] - On the approximation by nonlinear integral operators with generalized Lipschitz kernel over a locally compact abelian group, Comment. Math. Prace Mat. 34 (1995), 153-164.

[6] - On some linearly indexed families of submeasures, to appear in Atti del Convegno "Real Analysis and Measure Theory" (Ischia July 1-6, 1994) and in Atti Sem. Mat. Fis. Univ. Modena.

DIPARTIMENTO DI MATEMATICA

UNIVERSITÀ DEGLI STUDI

VIA VANVITELLI, 1

06123 PERUGIA, ITALY

E-mail: MATEVIN@IPGUNIV.BITNET
FACULTY OF MATHEMATICS AND COMPUTER SCIENCE ADAM MICKIEWICZ UNIVERSITY MATEJKI 48/49 60-769 POZNAŃ, POLAND 